\title{
Linking perception to decision point complexity for adaptive indoor wayfinding support
}

\author{
Laure De Cock $^{\mathrm{a}, *}$, Kristien Ooms ${ }^{\mathrm{a}}$, Nico Van de Weghe ${ }^{\mathrm{a}}$, Philippe De Maeyer ${ }^{\mathrm{a}}$ \\ ${ }^{a}$ Ghent University, Laure de Cock, laudcock.decock@ugent.be, Kristien Ooms, kristien.ooms@ugent.be, Philippe De Maeyer, \\ philippe.demaeyer@ugent.be \\ * Corresponding author
}

Keywords: Indoor wayfinding, Route communication, Space syntax, Isovist, Complexity

\begin{abstract}
:
Outdoors, navigation aids are widely used. Indoors, on the other hand, these systems are not yet common practice, because it requires another more elaborate positioning method and the environments are more complex. Especially in complex buildings, wayfinding can be challenging and induce a rise in cognitive load. To reduce cognitive load, indoor wayfinding aids have to be adapted to both the architecture of the building and the requirements of the users.
\end{abstract}

To facilitate this adaptation, first of all, the complexity of a building has to be quantified. Several methodologies are developed to this end and some of them have been proven to correlate well with wayfinding strategies of navigators, such as isovists and the space syntax theory. An isovist is the area in space which can be seen from a certain viewpoint. This isovist area can be drawn on a floorplan of a building, resulting in a polygon with several geometric properties (e.g. surface area, longest line of sight, compactness) that can be used to quantify the complexity in a certain point of a building. Rather than focussing on separate elements of space, the space syntax theory analyses how spaces relate to each other. The visibility graph analysis (VGA) combines both methodologies by putting a grid on the floorplan and drawing an isovist in every point of the grid. From all these points, measures such as the mean visual depth can be calculated, which quantify the integration of the points in the global building structure. Measures of isovists and VGA are often used in literature to link spatio-visual properties of buildings (e.g. museums and shopping centers) to exploratory movements of visitors. The architectural layout of a building has a significant impact on the cognitive load during navigation, but the demanded effort also depends on people's environmental perception. One of the factors that shape this perception are personal characteristics such as cultural background, familiarity and navigation capabilities. The quantification of decision point visibility with isovist measures or VGA on the one hand and the navigator's perception of the same decision point on the other hand are not necessarily equivalent. However, both have an influence on indoor movement and therefore, both should be included in a framework for an adaptive wayfinding aid.

This adaptation can be implemented in several components of a wayfinding system (e.g. the route planning by generating the least turn path instead of the shortest path). However, the route instructions convey the spatial information and are therefore the key to successful navigation. Adequate communication can accelerate the process of wayfinding and avoid uncertainties of the user. Moreover, the chosen presentation form of the instruction is decisive for the usability of the navigation system. A distinction can be made between maps and turn-by-turn instructions. Maps give a survey perspective of the environment by displaying the complete floorplan. That way, navigators can improve their cognitive map of the environment. However, most users of wayfinding aids in complex buildings have no interest in improving their mental map and prefer a maximum ease of wayfinding. Alternatively, turn-by-turn instructions can be given at every decision point in many forms (e.g. symbols, text, map, photo, 3D simulations). The current studies on route communication are limited to one type per route. However, because every decision point is different, the induced cognitive load is also different at these points. Therefore, route communication should be adapted at decision point level.

To be able to make an adaptive wayfinding system an online survey is executed to determine the best type of route instruction for every decision point (Figure 1). The case study building of the online survey is the iGent tower, a recently built (2015) office building in Ghent (Belgium). The architects designed the tower as a smart building (with several location sensors implemented in the ceiling), hence, it is well equipped for the development of a wayfinding system. During the survey, participants are guided along ten routes, based on video materials. For every new route, a different type of route instruction is shown whereby the order of both the routes and the types is randomised. At every decision point along the ten routes ( 52 in total) participants indicated how complex they found that decision point and how good they found that instruction type. The answers to the first question are compared to six isovist measures and one VGA measure (calculated with Isovists.org). The preliminary results indicate that several measures are correlated with the complexity ratings of the participants. However, a factor with a larger influence on the complexity ratings is the action participants had to take (e.g. go straight forward, turn left, change floors). Therefore, the complexity of a decision point depends both on the architecture and on the type of action navigators have to take. It can be concluded that both aspects should be considered in the instruction type selection of an adaptive wayfinding system. This conclusion is confirmed by the instruction type ratings. 

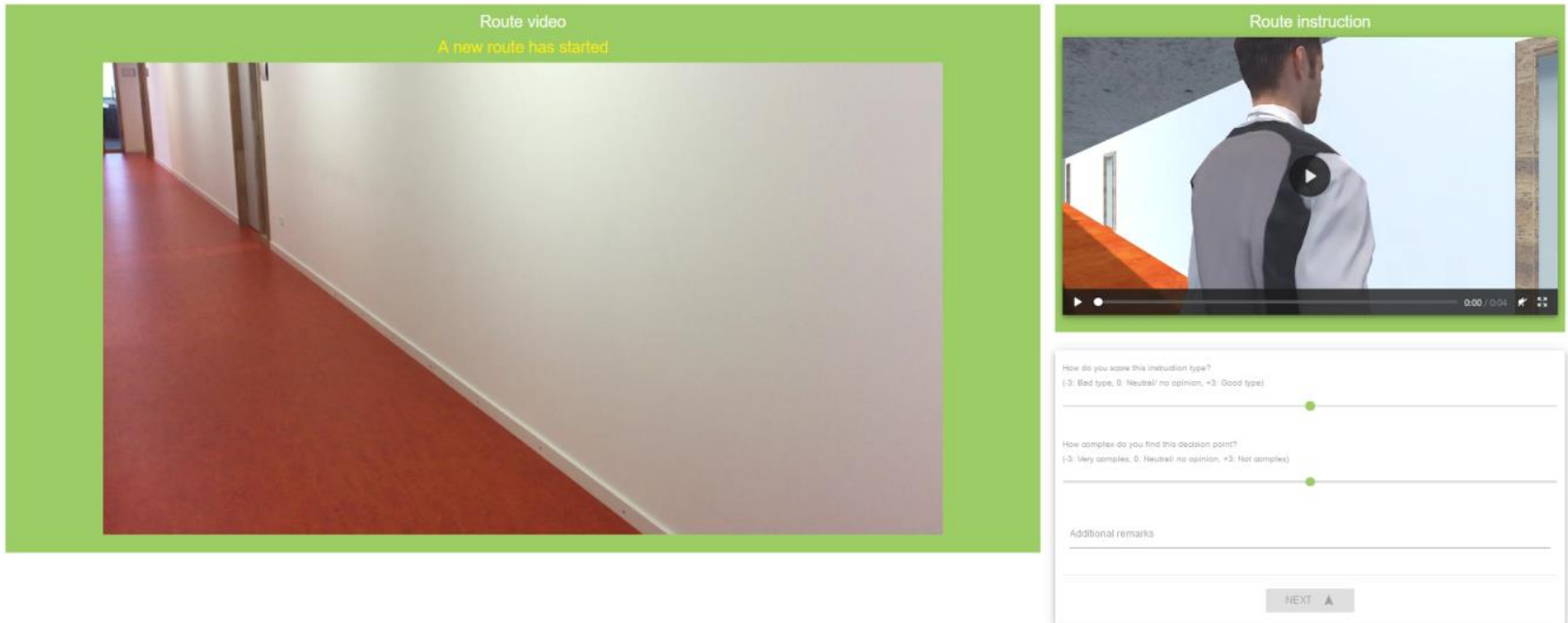

Figure 1: Online survey. 